\title{
Diagnostic model based on Raman spectra of normal, hyperplasia and prostate adenocarcinoma tissues in vitro
}

\author{
Ricardo Matias Lopes a , Landulfo Silveira Jr. ${ }^{\text {b, }}$, Marcos Augusto R.S. Silva ${ }^{\text {a }}$, \\ Kátia Ramos M. Leite ${ }^{\mathrm{c}}$, Carlos Augusto G. Pasqualucci ${ }^{\mathrm{d}}$ and Marcos Tadeu T. Pacheco ${ }^{\mathrm{b}}$ \\ ${ }^{a}$ Institute of Research and Development, Universidade do Vale do Paraíba, São José dos Campos, SP, \\ Brazil \\ ${ }^{\mathrm{b}}$ Group of Biomedical Engineering, Universidade Camilo Castelo Branco, São José dos Campos, SP, \\ Brazil \\ ${ }^{\mathrm{c}}$ Department of Urology, University of São Paulo Medical School, São Paulo, SP, Brazil \\ ${ }^{\mathrm{d}}$ Department of Pathology, University of São Paulo Medical School, São Paulo, SP, Brazil
}

\begin{abstract}
This study evaluated the use of Raman spectroscopy to identify the spectral differences between normal (N), benign hyperplasia $(\mathrm{BPH})$ and adenocarcinoma $(\mathrm{CaP})$ in fragments of prostate biopsies in vitro with the aim of developing a spectral diagnostic model for tissue classification. A dispersive Raman spectrometer was used with $830 \mathrm{~nm}$ wavelength and $80 \mathrm{~mW}$ excitation. Following Raman data collection and tissue histopathology (48 fragments diagnosed as N, 43 as BPH and 14 as $\mathrm{CaP}$ ), two diagnostic models were developed in order to extract diagnostic information: the first using PCA and Mahalanobis analysis techniques and the second one a simplified biochemical model based on spectral features of cholesterol, collagen, smooth muscle cell and adipocyte. Spectral differences between N, BPH and CaP tissues, were observed mainly in the Raman bands associated with proteins, lipids, nucleic and amino acids. The PCA diagnostic model showed a sensitivity and specificity of $100 \%$, which indicates the ability of PCA and Mahalanobis distance techniques to classify tissue changes in vitro. Also, it was found that the relative amount of collagen decreased while the amount of cholesterol and adipocyte increased with severity of the disease. Smooth muscle cell increased in BPH tissue. These characteristics were used for diagnostic purposes.
\end{abstract}

Keywords: Raman spectroscopy, prostate cancer, benign prostatic hyperplasia, Principal Components Analysis (PCA), Mahalanobis distance, biochemical model

\section{Introduction}

During the last century, the understanding of prostate diseases changed significantly due to screening of benign and malignant diseases. Benign prostatic hyperplasia (BPH) occurs only in the periurethral glands, particularly in the transition zone, and is characterized by stromal and epithelial hyperplasia [19]. It's occurrence is highly correlated with the reduction of androgens in aging males. Prostate adenocarcinoma $(\mathrm{CaP})$ can be presented in a variety of clinical forms, from asymptomatic, latent tumors to highly aggressive or metastasized tumors. These tumors usually originate from epithelial cells of the

\footnotetext{
*Corresponding author: Landulfo Silveira Jr., Group of Biomedical Engineering, Universidade Camilo Castelo Branco, Núcleo do Parque Tecnológico de São José dos Campos, Rod. Pres. Dutra, São José dos Campos, SP, Brazil 12247-004. Tel./Fax:+55 123905 4401; E-mails: landulfo.silveira@unicastelo.br, landulfo.silveira@gmail.com.
} 
peripheral zone of the gland [21]. In normal prostate tissue, low rate of cell proliferation is balanced with the low rate of apoptosis. When cell proliferation rates are on the rise, the cells begin to induce the production of TGF- $\beta$, a physiologic regulator of cell death, to counteract the proliferative activity of the prostate which maintains the equilibrium. The BPH begins with the disruption of this process [16].

Researchers have been seeking minimally invasive procedures that are able to provide molecular diagnostic information and allow for the real time analysis of the tissues' biochemistry at the cellular and molecular level. Optical spectroscopy can provide diagnostic information through the evaluation of tissue response to light excitation, since specific interactions are produced by the incident radiation in tissues [8]. Raman spectroscopy has been particularly promising due to the molecular information contained in the Raman spectra. The Raman technique evaluates the interaction of the incident monochromatic light with the molecules. This interaction causes a loss or a gain in the vibrational energy of molecules, which appears as bands at specific positions of the molecular group vibrations [22]. A Raman spectrum gives insight into the molecular composition of the sample and could provide diagnostic information for the pathology under study. This would be a viable alternative to the histopathology of $\mathrm{BPH}$ or $\mathrm{CaP}$ tissues, which is subjective and is in some cases associated with considerable inter-observer variations [1].

Several studies have confirmed the accuracy of the diagnostic technique based on Raman spectroscopy in various biological tissues such as the cervical lesions [1,22], gastric tissues [15,30], colon [20], thyroid [18], larynx [34], skin [2,17], bladder [6,10], among others. In prostate tissues, several studies indicated the diagnostic capability of various optical techniques [5,7,9,33].

Several studies have proposed the use of multivariate techniques such as Principal Components Analysis (PCA) and Discriminant Analysis (DA) in the development of diagnostic algorithms based on the information presented in the Raman spectra [5-7,9,34]. PCA has been employed for spectral classification in a variety of diseases such as atherosclerosis and neoplasias, among others $[9,10,13-15,17,20,26$, 27,34,35]. With PCA, one could separate the relevant spectral information which is dependent on the composition of the material analyzed, i.e., a few variables, from the random, uncorrelated variations.

PCA extracts the relevant information from the original data (A) and generates a new set of variables called principal components vectors (PCs) and scores $(\mathrm{S}): \mathrm{A}=\mathrm{PC} \times \mathrm{S}$. The PCs are related to the most significant variations across the spectra. The first PCs are related to the larger variation of the data and the last PCs carry only noise [31]. Discriminant analysis is used to separate samples with similar characteristics into well-defined groups or classes. The Mahalanobis distance is a very useful way to identify the similarities of a data set within the same group. Since it takes into account the covariance matrix, it is well suited for discriminating non-circular distribution [11,29]. Crow et al. employed Raman spectroscopy to identify and grade prostate adenocarcinoma in vitro using biopsy fragments, thereby building a diagnostic algorithm using the Principal Components Analysis (PCA) and Linear Discriminant Analysis (LDA) [6,9]. Stone et al. used Raman micro-spectrometry to examine prostate biopsies with BPH and $\mathrm{CaP}$ and to classify fragments employing PCA and LDA [9]. Crow et al. collected Raman spectra from tissue fragments obtained after transurethral prostate resection (TURP) with the aid of a fibre optic cable coupled to a Raman spectrometer. An algorithm was developed based on PCA and LDA for optical diagnosis and was evaluated using the sensitivity and specificity [7]. Crow et al. also used microRaman spectroscopy to study cultures of human prostate cancer cell lines and constructed an algorithm to classify the samples using PCA/LDA [5].

Raman spectroscopy is also capable of estimating the relative amount of selected biochemicals present in biological samples which may be used for diagnostic purposes $[12,23,33]$. The relative amount of 
such biochemicals can be predicted by linearly fitting the tissue spectra to the spectra of those biochemicals. Such biochemical information could be used for diagnostic purposes, by comparing the changes in the biochemicals with the disease stage measured through histopathology. The tissue identification and classification into specific classes could then be carried out by selecting a discriminator. Haka et al. proposed the diagnosis of breast cancer by calculating the amount of fat, collagen, cholesterol, cell nucleus and cytoplasm and calcium oxalate through Raman spectra and comparing this with the normal tissue [12]. Motz et al. used a Raman probe connected to a near-infrared Raman spectrometer to scan carotid arteries in vivo in order to gain insight into the biochemicals present in the sample, and correlated the plaque composition with the possible prevention of plaque vulnerability [23]. Stone et al. used Raman micro-spectrometry to analyze benign and malignant prostate and bladder fragments. They developed a biochemical model based on spectra of pure compounds such as actin, collagen, choline, triolein, oleic acid, cholesterol and DNA in order to assess the gross biochemical changes in each of the pathologies [33].

The aim of this work was to use dispersive near-infrared Raman spectroscopy to identify the main spectral differences between normal prostate $(\mathrm{N})$, benign prostatic hyperplasia $(\mathrm{BPH})$ and prostate adenocarcinoma $(\mathrm{CaP})$ tissue fragments in vitro. A diagnostic model based on the Raman spectra optical technique was developed using multivariate statistics (PCA) and discriminant analysis (Mahalanobis distance) to classify N, BPH and CaP spectra in one of the three histopathological groups. Also, the relative amount of cholesterol, collagen, smooth muscle cell and adipocyte was calculated by fitting a simplified biochemical model to each tissue type. The purpose of which was to assess the gross constitution of prostate tissue, searching for a correlation of the biochemical changes with the tissue histopathology.

\section{Materials and methods}

\subsection{Prostate tissue samples}

After protocol approval by a Research Ethics Committee, prostate gland specimens were obtained in three ways. Firstly, six normal prostate specimens (without malignant alterations confirmed by histopathology) were obtained from Autopsy Service of University of São Paulo, São Paulo, SP, Brazil, taken from young adult cadavers (up to 30 years of age) with less than $12 \mathrm{~h}$ of death. These specimens were sectioned transversely (approximately $5 \mathrm{~mm}$ ) in the posterior portion that corresponds to the peripherical zone, furnishing 48 fragments. Secondly, 12 benign prostatic hyperplasia specimens (approximately $20 \mathrm{~mm}$ ) were surgically removed through transuretral resection of the prostate (TURP) at Hospital Carlos Chagas, Guarulhos, SP, Brazil and sectioned transversely (5 $\mathrm{mm}$ fragments), furnishing 43 fragments. Thirdly, prostate adenocarcinoma specimens from four patients with confirmed neoplasia were surgically removed by radical prostatectomy procedures carried out at Hospital Sírio Libânes, São Paulo, SP, Brazil, furnishing 14 samples (approximately $5 \mathrm{~mm}$ ). The neoplasia was confirmed by a digital rectal examination and histological findings from Gleason's grade and tumor volume. This was an indication for the radical prostatectomy after persistent abnormal PSA levels. Moreover, all samples were snap-frozen and stored in liquid nitrogen $\left(-196^{\circ} \mathrm{C}\right)$ prior to Raman spectroscopy analysis.

At the end of the Raman spectra collection, the spot where the incident laser hit the fragment was marked with India ink and the sample fragment was fixed with $10 \%$ formalin, bottled and labelled. $\mathrm{N}$ and BPH samples were then sent to the Laboratory of Molecular Pathology (CEDAC) in Guarulhos, SP, Brazil for microscopic histopathological examination. The prostate gland samples were subjected to 
traditional histopathological processing, stained with $\mathrm{H} \& \mathrm{E}$ and analyzed under an optical microscope. Those sections containing N, BPH and CaP were then classified by a board-certified urological pathologist. The results were subjected to a spectral diagnosis using a discrimination model.

\subsection{Raman spectroscopy}

At the time of Raman data collection, the samples were moisturised with $0.9 \%$ saline and warmed to room temperature. Tissue fragments were cut into $3 \times 3 \mathrm{~mm}$ pieces and placed in an aluminium sample holder. Dispersive near-infrared Raman spectra were obtained using a table-top dispersive Raman spectrometer. Briefly, the Raman excitation was carried out by a semiconductor laser (Microlaser Systems Inc., model L4830S) operating at $830 \mathrm{~nm}$ wavelength and $80 \mathrm{~mW}$ output power. The laser hits the sample through a lens ( $26 \mathrm{~mm}$ focal length) at a $90^{\circ}$ excitation-collection geometry. The scattered light is collected by a set of lenses (100 mm focal length) which passes through a band rejection notch filter at $830 \mathrm{~nm}$ (Spectral Iridian Technologies, model PN-ZX 000080) that is placed in front of the entrance slit in order to block the Rayleigh scattering from the laser. The scattered light is then coupled to an imaging spectrograph (Chromex, model 2501S) that disperses and directs the light to a CCD camera (Princeton Instruments, model LN/CCD-1024-EHR1) and controller (Princeton Instruments, model ST130). Spectra were obtained with an integrating time of $50 \mathrm{~s}$ under a spectrometer resolution of about $10 \mathrm{~cm}^{-1}$. One spectrum for each tissue fragment was collected.

Raman shift calibration was performed by collecting the spectrum of naphthalene on the day of data collection. The known band and the respective pixel positions of naphthalene were fitted with a 3rd order polynomial. All spectra were then pre-processed prior to PCA. Basically, the fluorescence background was removed using a 5 th order polynomial fitted over the $800-1800 \mathrm{~cm}^{-1}$ spectral range and the cosmic rays were removed manually. Finally, each spectrum was normalized using the most intense band at around $1450 \mathrm{~cm}^{-1}$ and mean-centred.

\subsection{Diagnostic model based on Principal Components Analysis (PCA)}

After data pre-processing, spectra were separated in three groups according to the histopathology: N, BHP and CaP prostate tissues. A diagnostic model was developed using PCA and discriminant analysis for sample classification based upon the histopathology.

For the diagnostic model, principal components vectors (PC) and principal component scores (S) were calculated from all 105 spectra using PCA (Matlab 7.0, The Mathworks, Inc., USA) with the NIPALS algorithm [36] using a program developed elsewhere [28] for the Matlab platform. The PC carries information which represents the spectral variations of the dataset. The score S contains the intensity of each PC in the original spectrum. In order to identify which scores best discriminate between the histopathological groups, the non-parametric ANOVA test (significance level of 5\%) was applied to the scores of PC1-PC3.

The Mahalanobis distance is a useful way to determine the similarities between a set of values from one group of samples compared to another group and has been described as a multivariate equivalent of the confidence interval $[4,11]$. It compares the distance of an observed point to the points that comprise the modelled space. This space is configured during the modelling using the information provided by the knowledge of the sample composition [4,11]. The mean Mahalanobis distance line can then be drawn between the groups.

The relative amount of biochemicals present in the prostate fragments were estimated using the Raman spectra of cholesterol (from powder), collagen (from tendon), smooth muscle cell (from esophagus) and 
adipocyte (from perivascular fat). The last three were obtained from human post mortem bulk fragments. Spectra of the tendon and perivascular fat were compared to the spectra of collagen (spectral features of type I and III) and adipocyte (spectral features of triolein) [33] in literature. The relative amounts of these biochemicals were calculated by the least-square fitting of the basal spectra with the spectra of the tissue. This was achieved by solving the equation: $\mathrm{X}=\mathrm{C} \times \mathrm{SC}+\mathrm{E}$, where $\mathrm{X}$ is the original spectra, $\mathrm{SC}$ is the spectra of tissue constituents, $\mathrm{C}$ is the predicted concentration of the constituents and $\mathrm{E}$ is the residuals. This fitting assumes that residuals are minimized and the selected spectral components are the major components of tissue. The disadvantage of this fitting is that any colinearity in the selected components or absence of important basal compounds would skew the fit [33]. The non-negative constrained fitting was used, in order to avoid possible negative coefficients.

\section{Results}

The normalized mean Raman spectra of N, BPH and CaP fragments in the fingerprint region (800$1800 \mathrm{~cm}^{-1}$ ) are presented in Fig. 1. It was found that the Raman spectra of N, BPH and CaP prostate tissues have similar spectral features, however some bands have significantly different relative intensities. The main bands for all tissues are summarized in Table 1 [32]. Spectra of the normal prostate specimens have higher Raman peaks compared to $\mathrm{BPH}$ and $\mathrm{CaP}$ specimens at $861 \mathrm{~cm}^{-1}$ (amino-acids tyrosine and proline), $941 \mathrm{~cm}^{-1}$ (C-C chain from amino acids proline and valine and also proteins), $1002 \mathrm{~cm}^{-1}$ (phenylalanine ring), $1100 \mathrm{~cm}^{-1}$ (C-N bond of proteins and lipids) and $1663 \mathrm{~cm}^{-1}$ (amide I: $\mathrm{C}=\mathrm{O}$ bond of proteins; $\alpha$-helix conformation; $\mathrm{C}=\mathrm{C}$ bond of lipids). The prostate adenocarcinoma fragments showed more evident Raman bands at $1252 \mathrm{~cm}^{-1}$ (amide III: $\mathrm{C}-\mathrm{N}$ bond of collagen; cytosine and tyrosine; DNA), $1328 \mathrm{~cm}^{-1}\left(\mathrm{CH}_{3} / \mathrm{CH}_{2}\right.$ bond of collagen; polynucleotide chain), $1451 \mathrm{~cm}^{-1}\left(\mathrm{CH}_{2}\right.$ bending of proteins; $\mathrm{CH}_{2}$ deformation of lipids and proteins) and $1560 \mathrm{~cm}^{-1}$ (nucleic acids adenine and guanine). Some bands of BPH specimens showed intermediate intensities compared to the bands of the specimens $\mathrm{N}$ and $\mathrm{CaP}$.

In order to develop a diagnostic model of prostate tissue based on the spectral differences correlated with the histopathology, the PCA (data reduction) and the Mahalanobis distance (discriminant analysis) techniques were employed. ProRaman software [28] and Matlab was used for the PCA calculation using

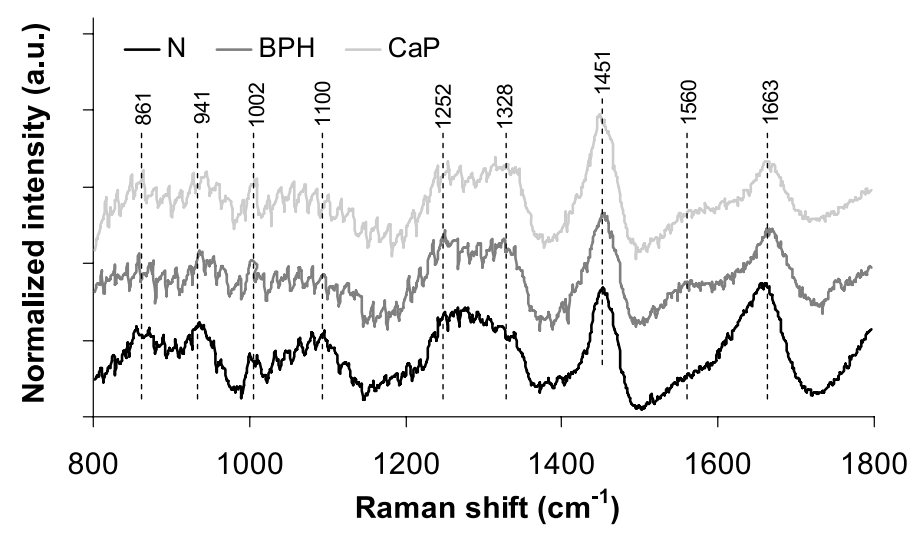

Fig. 1. Mean Raman spectra of normal prostate tissue (N), benign prostatic hyperplasia (BPH) and prostate adenocarcinoma (CaP) obtained from tissue fragments in vitro. Laser power: $80 \mathrm{~mW}$, wavelength: $830 \mathrm{~nm}$, spectrometer resolution: $10 \mathrm{~cm}^{-1}$. 
Table 1

Molecular vibration assignments for the Raman bands of prostate tissues [32]

\begin{tabular}{ll}
\hline Band $\left(\mathrm{cm}^{-1}\right)$ & \multicolumn{1}{c}{ Functional groups and molecular vibration assignment } \\
\hline 861 & Ring breathing of tyrosine; $\mathrm{C}-\mathrm{C}$ stretching of proline \\
941 & $\mathrm{C}-\mathrm{C}$ stretching of proline and valine and protein backbone ( $\alpha$-helix conformation); glycogen \\
1002 & Symmetric ring breathing of phenylalanine \\
1100 & $\mathrm{C}-\mathrm{N}$ stretching of proteins (and lipids in lesser degree) \\
1252 & Amide III: collagen $\left(\mathrm{CH}_{2}\right.$ wagging, $\mathrm{C}-\mathrm{N}$ stretch); pyrimidine bases $(\mathrm{C}, \mathrm{T})$ \\
1328 & $\mathrm{CH}_{3} / \mathrm{CH}_{2}$ wagging of collagen; polynucleotide chain (DNA-purine bases) \\
1451 & $\mathrm{CH}_{2}$ bending of proteins; $\mathrm{CH}_{2}$ deformation (lipids and proteins) \\
1560 & Adenine, guanine \\
1663 & Amide $\mathrm{I}(\mathrm{C}=\mathrm{O}$ stretching of proteins, $\alpha$-helix conformation $) ; \mathrm{C}=\mathrm{C}$ stretching of lipids \\
\hline
\end{tabular}

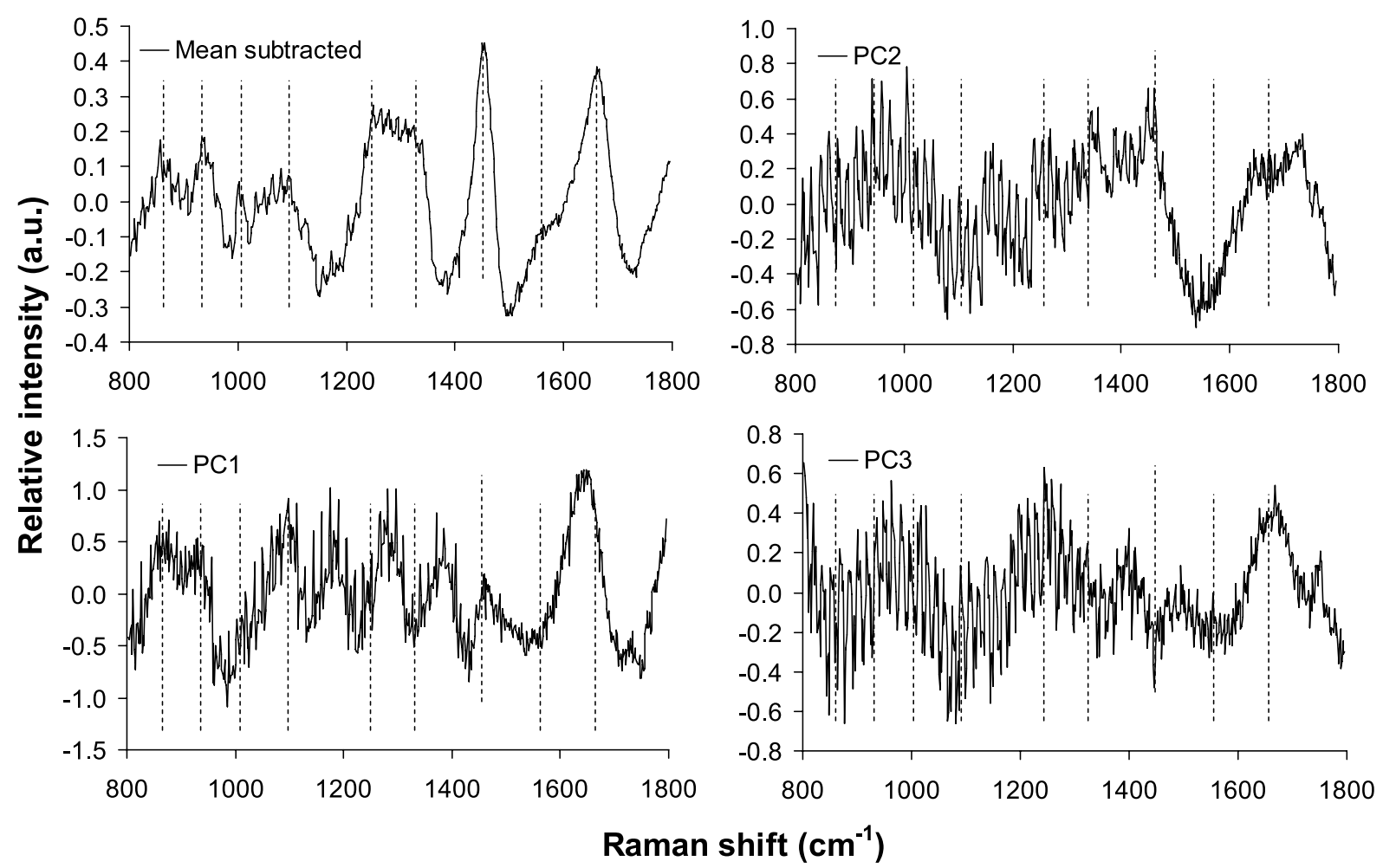

Fig. 2. Average spectra of the dataset which was subtracted from each spectrum before PCA calculations and first three principal components vectors (PC1, PC2 and PC3) with the labeled Raman bands.

the mean-centered, mean-subtracted spectra of $\mathrm{N}, \mathrm{BPH}$ and $\mathrm{CaP}$ tissues. It was found that the principal components vectors PC1, PC2 and PC3 have a relevance of approximately 23, 9 and 5\%, respectively, after mean subtracting.

Figure 2 shows the average spectrum (that was subtracted for PCA) and the first three principal components vectors of the prostate samples. These vectors resemble Raman spectra, with positive and negative bands in positions similar to those found in the spectra of tissues, as demonstrated by the corresponding labelled bands. 

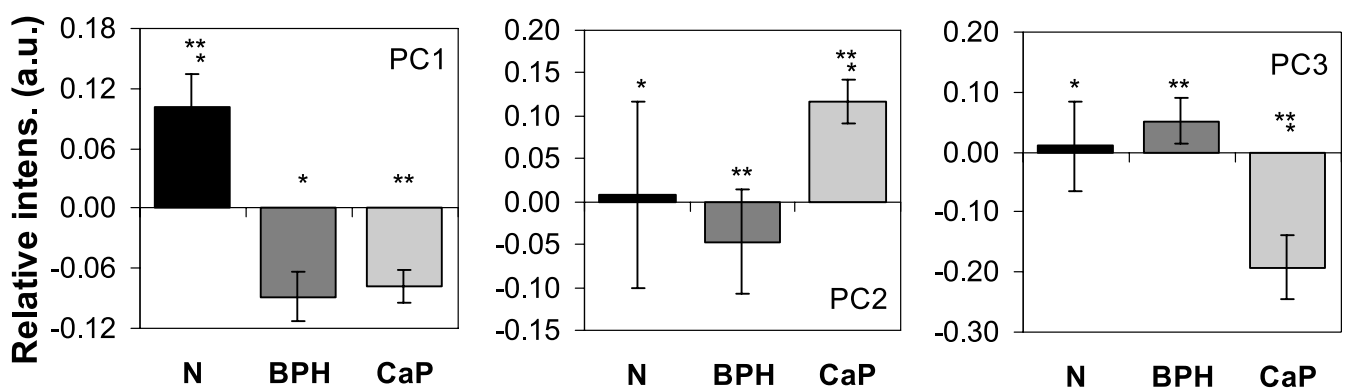

Fig. 3. Mean and standard deviation intensity of the principal components scores from PC1, PC2 and PC3 vectors. ${ }^{*}$ and ${ }^{* *}$ indicate $p<0.05$ calculated with parametric ANOVA comparing $\mathrm{N}, \mathrm{BPH}$ and $\mathrm{CaP}$ in the selected $\mathrm{PCs}$.

In order to find the model's principal component that best differentiates between the tissue types, the non-parametric ANOVA test at a 5\% level of significance was applied to the first three principal components scores (Fig. 3). It was found that PC1 could better differentiate $\mathrm{N}$ tissue from BPH and $\mathrm{CaP}$ tissues $(p<0.001)$. PC2 could better differentiate $\mathrm{N}$ from CaP tissue and $\mathrm{BPH}$ from CaP tissue $(p<0.01)$. PC3 could better differentiate $\mathrm{N}$ from CaP tissue and $\mathrm{BPH}$ from $\mathrm{CaP}$ tissue $(p<0.001)$. PC scores were plotted in pairs $(\mathrm{PC} 1 \times \mathrm{PC} 2, \mathrm{PC} 1 \times \mathrm{PC} 3$ and $\mathrm{PC} 2 \times \mathrm{PC} 3)$ in Fig. 4, allowing for the visualization of the grouped data. The Mahalanobis distance method was then applied to these PC pairs to discriminate and consequently classify the spectra according to the distance of each data point to the group with similar information. It is presented as a surface that separates the data into selected classes. Therefore the PC1 $\times$ PC3 was well suited to distinguish between the spectra of the three tissue types with the diagnostic's maximum sensitivity and specificity values shown in Table 2.

The relative amount of cholesterol, collagen, smooth muscle cell and adipocyte for each tissue type was obtained from the Raman spectra. Figure 5 shows the normalized spectra of basal compounds used in the fitting (taken with $100 \mathrm{~s}$ exposure time). Figure 6 presents the relative amount of the selected biochemical as a function of tissue type, calculated by solving the linear equation of the tissue spectrum with the basal spectra. It was found that the amount of cholesterol was virtually zero in normal samples and increased with disease status $(p<0.05)$. BPH samples showed an increased amount of smooth muscle cell compared to $\mathrm{N}$ and CaP samples $(p<0.05)$. Collagen content was found to be increased in the $\mathrm{N}$ samples as compared to the diseased tissues $(p<0.05)$ where it was reduced. Adipocytes increased in $\mathrm{CaP}$ samples and were virtually zero in $\mathrm{N}$ and BHP samples $(p<0.05)$.

Figure 7 shows the relative amount of smooth cell versus collagen for the three prostatic tissue types. The correlation of the amount of smooth cell versus collagen, accounting for the entire dataset (three tissue types), had a coefficient $r=-0.754$. When considering only $\mathrm{N}$ and BPH data, the correlation coefficient was $r=-0.906$. The relative amount of collagen was reduced in hyperplastic samples, although the amount of smooth cells increased. The $\mathrm{CaP}$ samples showed reduced amount of collagen and smooth cells. Figure 7 also shows the Mahalanobis classification surface applied to distinguish between $\mathrm{N}, \mathrm{BPH}$ and $\mathrm{CaP}$ tissue using the relative amount of collagen and smooth cell, with a high tissue differentiation capability (sensitivity and specificity higher than $90 \%$ for the CaP, Table 2 ).

\section{Discussion}

Morphologically, the prostate gland contains glandular epithelium, smooth muscle, stroma and a fibrous capsule (prostate capsule). Biochemically, these tissues are formed by cells which, in turn, are 

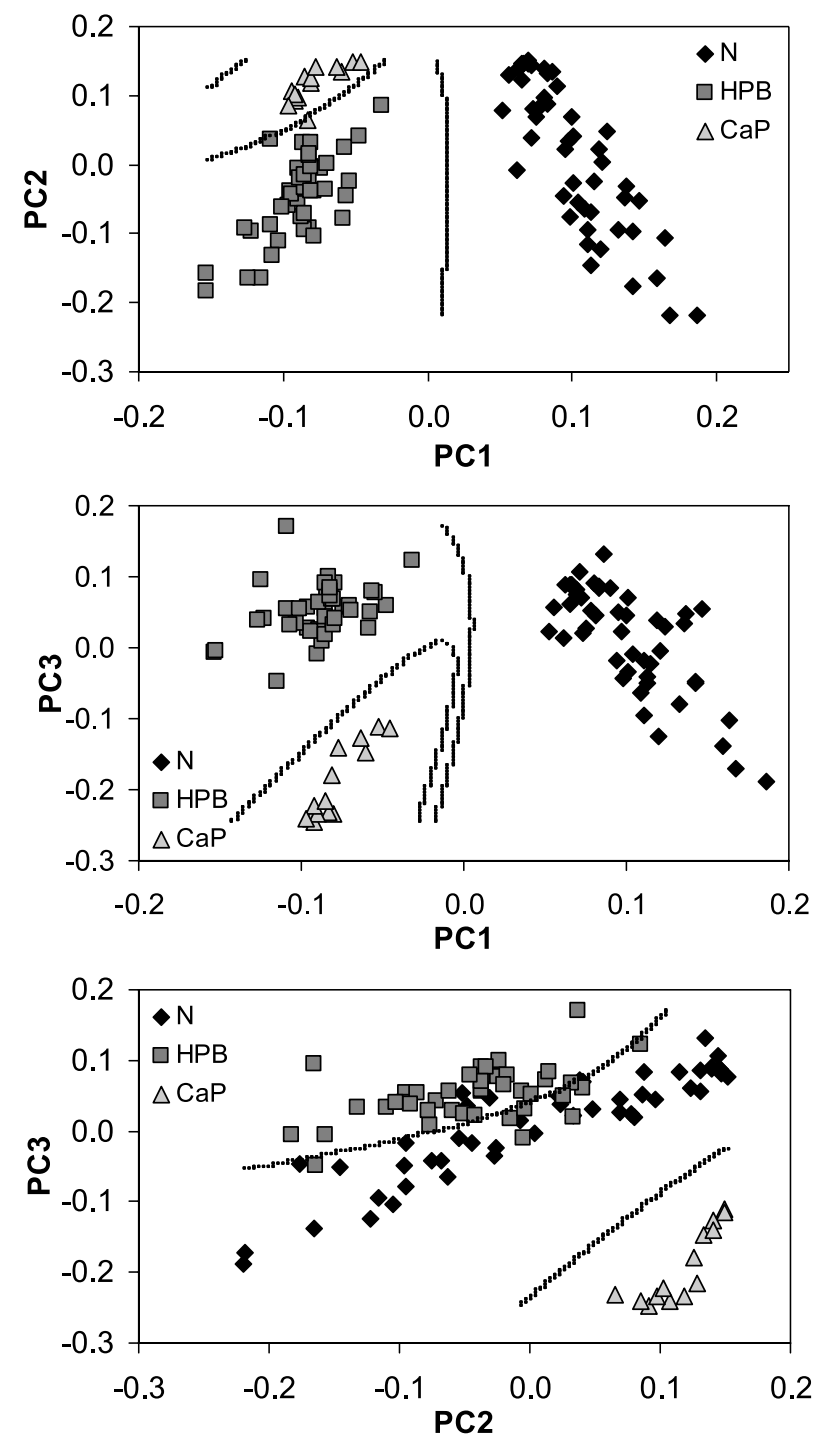

Fig. 4. Scatter plot of pairs of the first three principal components score $(\mathrm{PC} 1 \times \mathrm{PC} 2, \mathrm{PC} 1 \times \mathrm{PC} 3$ and $\mathrm{PC} 2 \times \mathrm{PC} 3)$ for the normal prostate $(\mathrm{N})$, benign prostatic hyperplasia $(\mathrm{BPH})$ and prostate adenocarcinoma $(\mathrm{CaP})$ samples. The curves represent the Mahalanobis classification surfaces.

composed of carbohydrates, lipids, proteins (collagen), amino acids and nucleic acids (DNA/RNA). Malignant transformation can occur in both normal-size (not hyperplastic) prostates and in those that have already undergone some degree of hyperplasia, but severe BPH may not develop malignancy. Biochemical alterations found in $\mathrm{BPH}$ and $\mathrm{CaP}$ tissues are due to cellular changes that occur. These alterations could be detected through Raman spectroscopy, by observing the variations of the intensities of peaks corresponding to the vibrations of chemical bonds of these molecules.

The dispersive Raman spectroscopy has been used as a methodology for optical diagnosis of benign and malignant prostate alterations. The major differences in the bands of prostate adenocarcinoma compared to other tissues ( $\mathrm{N}$ and $\mathrm{BPH}$ ) correspond to bands of structural proteins (mainly type IV collagen), 
Table 2

Sensitivity and specificity diagnostic values for the PCA and biochemical fitting models of prostate tissues in vitro

\begin{tabular}{lrrrrr}
\hline Histopatology & \multicolumn{5}{c}{ PCA diagnosis $(\mathrm{PC} 1 \times$ PC3) } \\
\cline { 2 - 6 } & $\mathrm{N}$ & $\mathrm{BPH}$ & $\mathrm{CaP}$ & Sensitivity $(\%)$ & Specificity $(\%)$ \\
\hline $\mathrm{N}(n=48)$ & 48 & 0 & 0 & 100 & 100 \\
$\mathrm{BPH}(n=43)$ & 0 & 43 & 0 & 100 & 100 \\
$\mathrm{CaP}(n=14)$ & 0 & 0 & 14 & Sensitivity $(\%)$ & Specificity $(\%)$ \\
\cline { 2 - 5 } & $\mathrm{N}$ & $\mathrm{BPH}$ & $\mathrm{CaP}$ & 88 & 87 \\
$\mathrm{~N}(n=48)$ & 41 & 7 & 0 & 93 & 97 \\
$\mathrm{BPH}(n=43)$ & 2 & 38 & 3 & 13 & \\
$\mathrm{CaP}(n=14)$ & 0 & 1 & & & \\
\hline
\end{tabular}

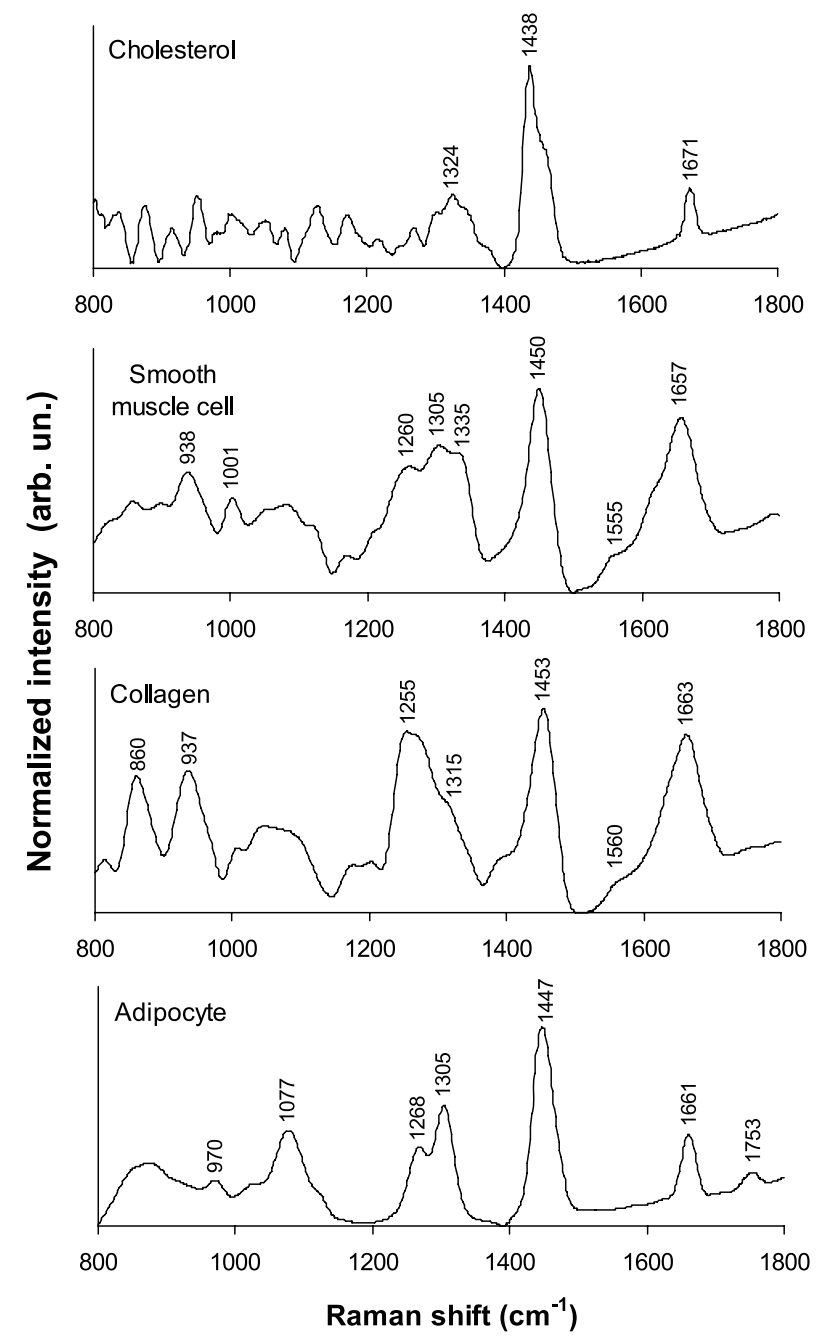

Fig. 5. Normalized Raman spectra of the basal compounds used in the biochemical model of prostate tissues. Excitation wavelength: $830 \mathrm{~nm}$, laser power: $100 \mathrm{~mW}$, integration time: $100 \mathrm{~s}$. 

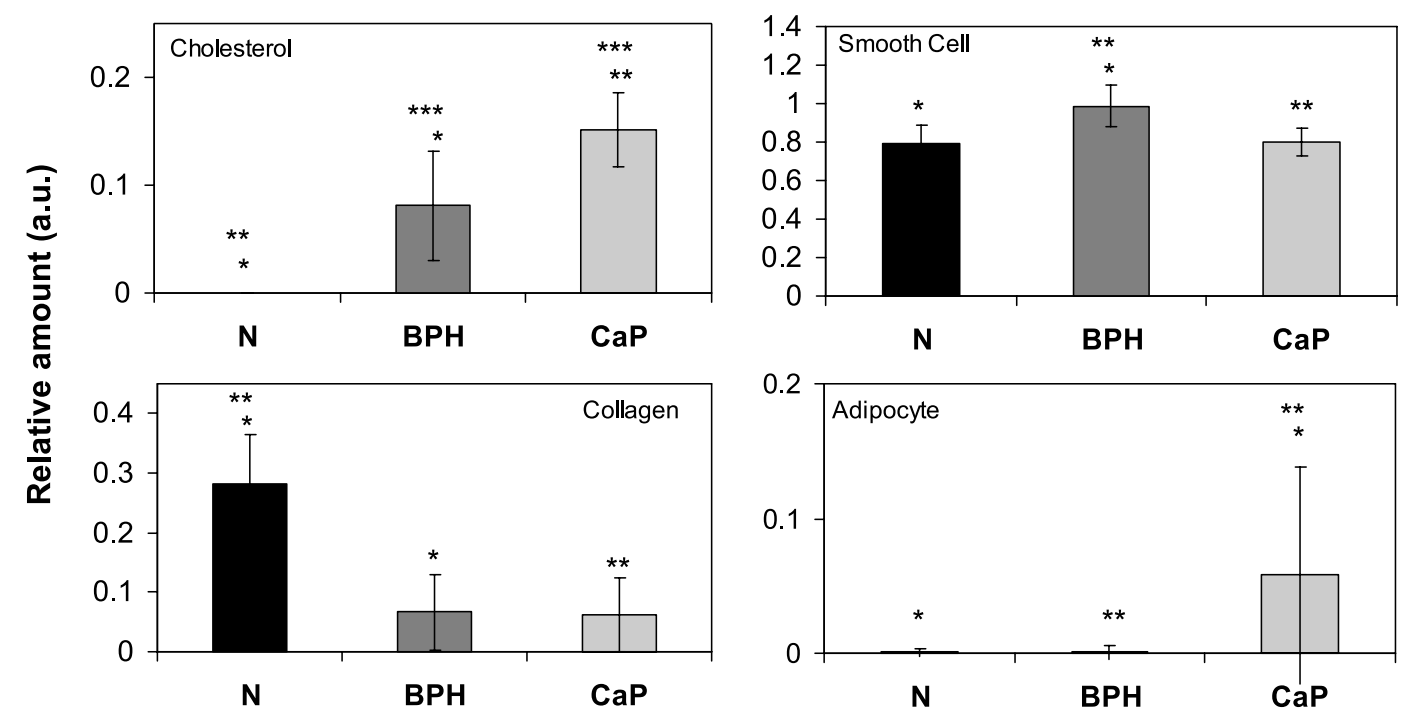

Fig. 6. Relative amount of each biochemical (cholesterol, smooth cell, collagen and adipocyte) calculated by fitting the basal spectra in the prostate tissue spectra. ${ }^{*},{ }^{* *}$ and ${ }^{* * *}$ indicate $p<0.05$ calculated with parametric ANOVA comparing N, BPH and $\mathrm{CaP}$ in the selected basal compounds.

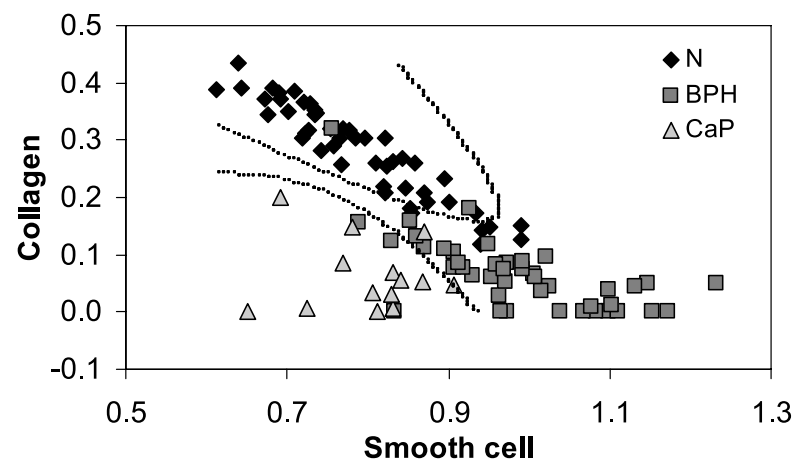

Fig. 7. Plot of the relative amount of smooth muscle cell versus collagen for the three prostatic tissue types. The correlation of the smooth cell versus collagen, considering the whole dataset was $r=-0.754$ and considering only N and BPH was $r=-0.906$. The lines represent the Mahalanobis classification surfaces.

cellular proteins and nucleic and amino acids (tyrosine, proline, valine, phenylalanine) [32]. The spectra of normal prostates and benign hyperplasia (BPH) partly resemble each other due to the biochemical similarities in the two tissues. The BPH differs from normal cells by the presence of hyperplasia, without malignancy features.

A diagnostic algorithm based on PCA and Mahalanobis distance was developed. The principal component vectors carry important information regarding the biochemical elements which can be related to bands present in the tissues. It was observed that PC1 showed positive bands at 861, 941, 1100, 1451 and $1663 \mathrm{~cm}^{-1}$ and negative bands at 1252 and $1328 \mathrm{~cm}^{-1}$ while PC 3 showed positive bands at 1252 and $1663 \mathrm{~cm}^{-1}$ and negative bands at 1100,1451 and $1560 \mathrm{~cm}^{-1}$. These bands are related to normal and diseased tissues and therefore represent the main variations in the dataset. These first three PC vectors were able to provide important information to differentiate between the Raman spectra of normal, BPH and 
$\mathrm{CaP}$ specimens, which was confirmed by the ANOVA test. The discriminant analysis based on Mahalanobis distance applied to the scores of PC1, PC2 and PC 3 which allowed for the separation of spectra according to the distance between the histopathological groups. The greater differentiation was obtained from PC1 $\times$ PC 3 with a sensitivity and specificity of $100 \%$ for BPH and CaP samples.

Also, a simplified biochemical model was presented, based on the relative amount of cholesterol, smooth muscle cell, collagen (proteins) and adipocytes (lipids), which have been shown to be responsible for the spectral differences in the BHP and CaP samples [33]. Results showed that collagen was increased in samples of $\mathrm{N}$ but reduced in $\mathrm{BPH}$ and $\mathrm{CaP}$ samples. Smooth cell content was increased in $\mathrm{BPH}$ samples. Adipocytes and cholesterol increased in $\mathrm{CaP}$ samples and reduced in $\mathrm{N}$ prostate samples. Collagen is a component of the basal membrane of the epithelium and with cellular multiplication in $\mathrm{BPH}$ and $\mathrm{CaP}$ samples, diminishing of cellular adhesion occurs. The increase of smooth cell (mainly actin) could be explained by the cellular proteins, due to an increase in the volume of BPH. With reference to adipocytes, the CaP samples showed a higher intensity than $\mathrm{N}$ and $\mathrm{BPH}$ samples, suggesting that $\mathrm{CaP}$ tissue has relatively more cell fat than $\mathrm{BPH}$ tissue due to a higher metabolic activity in $\mathrm{CaP}$ tissue. This could be explained by the fact that lipids sustain cellular growth in a tumour and by them being a part of the lipoproteic membrane. In the case of cholesterol, the intense cell multiplication and consequent cellular necrosis presented in $\mathrm{CaP}$ tissue induces the formation of cholesterol crystals in the extracellular matrix [33].

The Raman bands occurring in a specific region of the spectrum can arise from scattering of multiple types of macromolecules and it is not easy to distinguish if the spectral differences among samples are due to a specific type of macromolecule, mainly because bands superimpose on each other. Thus a prior knowledge of tissue biochemistry and composition is needed in order to correctly correlate the Raman bands that occur in malignant changes compared to the ones in benign tissues. Therefore the identification of the main bands of such biochemicals in the Raman spectra is important for the development of a reliable Raman diagnosis system. The use of a simplified model, based on the Raman contribution of a few important compounds, would ease the analysis.

Crow et al. showed that there are differences between the peaks of DNA of normal and tumoural tissue from the urinary bladder. Also mentioned, was that possible spectral differences may be present in other tissues and could be evaluated with Raman spectroscopy [6]. Crow et al. found an increase in the Raman bands of nucleic acids and decrease in the glycogen in CaP tissue compared to BPH tissue in vitro, with sensitivity and specificity ranging from 81 to $94 \%$ and between 92 and 100\%, respectively [9]. Crow et al. observed more intense Raman bands in the positions in the phenylalanine ring, amide III: $\mathrm{C}-\mathrm{C}$ stretching of proteins, $\mathrm{CH}_{2}$ twisting of collagen and lipids and the amide I band of proteins of prostate CaP tissues in vitro [7], which were similar to those described here. Still in 2005, the same author demonstrated that the Raman peaks related to nucleic acids showed lower concentrations in hormoneindependent cells (poorly differentiated) compared to androgen-dependent cells (well differentiated) in cell lines of prostate adenocarcinoma in vitro [5]. Stone et al. provided an estimation of the biochemistry associated with urological diseases and observed an increase in Raman bands of DNA, actin, choline and collagen in the prostate malignant tissue and reduction of beta-carotene, lycopene and glycogen in the same pathology [33].

In terms of tissue histochemistry, studies suggest that the growth of certain tumours is dependent on maintaining sufficient lipid levels and that the lipid-mobilizing effect of the tumour may be necessary to sustain tumour growth [25]. Also, the presence of cholesterol crystals in CaP tissue is common and could be a characteristic of high-grade lesions. For proteins, studies in oral tissue observed a progressive loss of continuity in the expression of collagen IV and laminin as tissues progressed from dysplasias 
to metastatic nodules [24]. This relation is important, as more aggressive carcinomas have an increased ability to produce enzymes that degrade basal membrane components, consequently hindering new protein synthesis [24]. Also, the presence of lower protein in CaP tissue, presented in $1663 \mathrm{~cm}^{-1}$ peak, may be explained by decrease of the glutathione $S$ transferase. These proteins function to protect the DNA from oxygen free radicals, impeding the formation of DNA adducts and consequently diminishing genetic errors. Recent studies have demonstrated a loss in the expression of this protein in $\mathrm{CaP}$ tissue development, leading to the action of environmental carcinogens in the prostate epithelial cells [28].

In this study, a diagnostic algorithm for tissue differentiation based on PCA and Mahalanobis distance resulted in sensitivity and specificity of $100 \%$ for $\mathrm{BPH}$ and $\mathrm{CaP}$. Also the fitted biochemical model showed a high sensitivity and specificity using the relative amount of smooth cell and collagen as a differentiator. Due to the small amount of samples of $\mathrm{CaP}$ tissue, a bias was present in the dataset. However, the results are of great value because it presents a possibility to distinguish benign hyperplasia from malignant lesion, and a possible evaluation of conditions such as inflammation (prostatitis), which is a study under way. Another important observation refers to the fact that normal prostate samples were obtained from cadavers with a time of death lower than $12 \mathrm{~h}$, leading to a possible interference of the cellular lysis and other molecular changes. To minimize this bias, normal prostate tissue should be analyzed ex vivo.

The research in this field would be directed for use during surgical procedures in order to help surgeons define the safe margin. This technique may be particularly useful when investigating patients with elevated PSA. If there is an indication for prostate biopsy, Raman spectroscopy could benefit the evaluation of biopsies without the bias of inter-observer assessment. The intention is to develop a diagnostic algorithm that includes all $\mathrm{CaP}$ grades and inflammation in software capable of classifying the various diseases, helping the pathologist to diagnose these changes rapidly and cost-effectively in real time.

\section{Conclusion}

Raman spectroscopy showed spectral differences in the biochemical composition of normal, benign hyperplasia and adenocarcinoma prostate tissues in vitro, especially in the Raman bands related to lipids, proteins and nucleic acids. Such differences were used to develop a diagnostic algorithm for the above conditions via an algorithm based on PCA and discriminant analysis by Mahalanobis distance. The proposed algorithms for prostate lesion samples have been classified in three histological groups with sensitivity and specificity of $100 \%$. It has been shown that PCA associated to Mahalanobis distance is an effective method to diagnose fragments of prostate lesions, opening the way for a Raman use ex vivo and in vivo in clinical environment. Also, the biochemical model using the Raman spectra of bulk samples showed that the relative amount of collagen reduced with disease severity and the amount of cholesterol, adipocyte and smooth cell increased from $\mathrm{N}$ to BPH and CaP samples. The amount of smooth cell and collagen could be used to differentiate disease status with high sensitivity and specificity.

\section{Acknowledgements}

L. Silveira Jr. thanks CNPq (Conselho Nacional de Desenvolvimento Científico e Tecnológico) for the Productivity Fellowship (305610/2008-2) and FAPESP (Fundação de Amparo à Pesquisa do Estado de São Paulo) for the Research Grant (2009/01788-5). 


\section{References}

[1] W.C. Allsbrook Jr., K.A. Mangold, W.C. Allsbrook, M.H. Johnson, R.B. Lane, C.G. Lane, M.B. Amin, D.G. Bostwick, P.A. Humphrey, E.C. Jones, V.E. Reuter, W. Sakr, I.A. Sesterhenn, P. Troncoso, T.M. Wheeler and J.I. Epstein, Interobserver reproducibility of Gleason grading of prostatic carcinoma: urologic pathologists, Human Pathology 32 (2001), 74-80.

[2] B. Bodanese, L. Silveira, R. Albertini, R.A. Zângaro and M.T.T. Pacheco, Differentiating normal and basal cell carcinoma human skin tissues in vitro using dispersive Raman spectroscopy: a comparison between principal components analysis and simplified biochemical models, Photomedicine and Laser Surgery 28 (2010), S119-S127.

[3] A. Chung, S. Wachsmann-Hogiu, T. Zhao, Y. Xiong, A. Joseph and D.L. Farkas, Advanced optical imaging requiring no contrast agents - a new armamentarium for medicine and surgery, Current Surgery 62 (2005), 365-370.

[4] E.J. Ciaccio, S.M. Dunn and M. Akay, Biosignal pattern recognition and interpretation systems. Part 3 of 4: methods of classification, IEEE Engineering in Medicine Biology Magazine 13 (1994), 129-135.

[5] P. Crow, B. Barrass, C. Kendall, M. Hart-Prieto, M. Wright, R. Persad and N. Stone, The use of Raman spectroscopy to differentiate between different prostatic adenocarcinoma cell lines, British Journal of Cancer 92 (2005), 2166-2170.

[6] P. Crow, C. Kendall, A. Ritchie, M. Wright and N. Stone, Evaluation of Raman spectroscopy to provide a real time, optical method for discrimination between normal and abnormal tissue in the bladder, European Urology Supplements 1 (2002), 80.

[7] P. Crow, A. Molckovsky, N. Stone, J. Uff, B. Wilson and L.M. Wongkeesong, Assessment of fiberoptic near-infrared Raman spectroscopy for diagnosis of bladder and prostate cancer, Urology 65 (2005), 1126-1130.

[8] P. Crow, N. Stone, C.A. Kendall, R.A. Persad and M.P. Wright, Optical diagnostics in urology: current applications and future prospects, British Journal of Urology International 92 (2003), 400-407.

[9] P. Crow, N. Stone, C.A. Kendall, J.S. Uff, J.A.M. Farmer, H. Barr and M.P.J. Wright, The use of Raman spectroscopy to identify and grade prostatic adenocarcinoma in vitro, British Journal of Cancer 89 (2003), 106-108.

[10] B.W.D. de Jong, T.C. Bakker Schut, K.P. Wolffenbuttel, J.M. Nijman, D.J. Kok and D.J. Puppels, Identification of bladder wall layers by Raman spectroscopy, Journal of Urology 168 (2002), 1771-1778.

[11] R. de Maesschalck, D. Jouan-Rimbaud and D.L. Massart, The Mahalanobis distance, Chemometrics Intelligent Laboratory Systems $\mathbf{5 0}$ (2000), 1-18.

[12] A.S. Haka, Z. Volynskaya, J.A. Gardecki, J. Nazemi, J. Lyons, D. Hicks, M. Fitzmaurice, R.R. Dasari, J.P. Crowe and M.S. Feld, In vivo margin assessment during partial mastectomy breast surgery using Raman spectroscopy, Cancer Research 66 (2006), 3317-3322.

[13] E.B. Hanlon, I. Itzkan, R.R. Dasari, M.S. Feld, R.J. Ferrante, A.C. Mckee, D. Lathi and N.W. Kowall, Near-infrared fluorescence spectroscopy detects Alzheimer's disease in vitro, Photochemistry and Photobiology 70 (1999), $236-242$.

[14] J. Hutchings, C. Kendall, B. Smith, N. Shepherd, H. Barr and N. Stone, The potential for histological screening using a combination of rapid Raman mapping and principal component analysis, Journal of Biophotonics 2 (2009), 91-103.

[15] T. Kawabata, T. Mizuno, S. Okazaki, M. Hiramatsu, M. Setoguchi, H. Kikuchi, M. Yamamoto, Y. Hiramatsu, K. Kondo, M. Baba, M. Ohta, K. Kamiya, T. Tanaka, S. Suzuki and H. Konno, Optical diagnosis of gastric cancer using near-infrared multichannel Raman spectroscopy with a 1064-nm excitation wavelength, Journal of Gastroenterology 43 (2008), 283290.

[16] N. Kyprianou, H. Tu and S.C. Jacobs, Apoptotic versus proliferative activities in human benign prostatic hyperplasia, Human Pathology 27 (1996), 668-675.

[17] C.H. Lieber, S.K. Majumder, D.L. Ellis, D.D. Billheimer and A. Mahadevan-Jansen, In vivo nonmelanoma skin cancer diagnosis using Raman microspectroscopy, Lasers in Surgery and Medicine 40 (2008), 461-467.

[18] M. Manfait, P. Lamaze, H. Lamfarraj, M. Pluot and G.D. Sockalingum, Diagnosis and prognosis of tissue pathologies by Raman microspectroscopy: an application to human thyroid tumours, Proceedings SPIE 3918 (2000), 53-160.

[19] J.E. McNeal, Origin and evolution of benign prostatic enlargement, Investigative Urology 15 (1978), 340-345.

[20] A. Molckovsky, W.K.L.M. Song, M.G. Shim, N.E. Marcon and B.C. Wilson, Diagnostic potential of near-infrared Raman spectroscopy in the colon? Differentiating adenomatous from hyperplastic polyps, Gastrointestinal Endoscopy 57 (2003), 396-402.

[21] L.B. Mora, R. Buettner, N. Ahmad, Y. Bassel, R. Jove and J.D. Seigne, Prostate adenocarcinoma: cellular and molecular abnormalities, Cancer Control 8 (2001), 551-561.

[22] L.M. Moreira, L. Silveira Jr., F.V. Santos, J.P. Lyon, R. Rocha, R.A. Zângaro, A.B. Villaverde and M.T.T. Pacheco, Raman spectroscopy: A powerful technique for biochemical analysis and diagnosis, Spectroscopy International Journal 22 (2008), 1-19.

[23] J.T. Motz, M. Fitzmaurice, A. Miller, S.J. Gandhi, A.S. Haka, L.H. Galindo, R.R. Dasari, J.R. Kramer and M.S. Feld, In vivo Raman spectral pathology of human atherosclerosis and vulnerable plaque, Journal of Biomedical Optics 11 (2006), 0210031 . 
[24] H.D. Mulligan and M.J. Tisdale, Effect of the lipid-lowering agent bezafibrate on tumor growth rate in vivo, British Journal of Cancer 64 (1991), 1035-1038.

[25] W.G. Nelson, A.M. de Marzo and T.L. DeWeese, The molecular pathogenesis of prostate cancer: implications for prostate cancer prevention, Urology 57 (2001), 39-45.

[26] A. Nijssen, T.C. Bakker, F. Schut, Heule, P.J. Caspers, D.P. Hayes, M.H.A. Neumann and G.J. Puppels, Discriminating basal cell carcinoma from its surrounding tissue by Raman spectroscopy, Journal of Investigative Dermatology 119 (2002), 64-69.

[27] G.V. Nogueira, L. Silveira, A.A. Martin, R.A. Zângaro, M.T. Pacheco, M.C. Chavantes and C.A. Pasqualucci, Raman spectroscopy study of atherosclerosis in human carotid artery, Journal of Biomedical Optics 10 (2005), 031117.

[28] A.R. Paula Jr., L. Silveira and M.T.T. Pacheco, ProRaman: a program to classify Raman spectra, Analyst 134 (2009), $1203-1207$.

[29] J. Saade, M.T.T. Pacheco, M.R. Rodrigues and L. Silveira, Identification of hepatitis C in human blood serum by nearinfrared Raman spectroscopy, Spectroscopy International Journal 22 (2008), 387-395.

[30] M.G. Shim, L.M.W.K. Song, N.E. Marcon and B.C. Wilson, In vivo near-infrared Raman spectroscopy: demonstration of feasibility during clinical gastrointestinal endoscopy, Photochemistry and Photobiology 72 (2000), 146-150.

[31] L. Silveira, S. Sathaiah, R.A. Zângaro, M.T.T. Pacheco, M.C. Chavantes and C.A.G. Pasqualucci, Correlation between near-infrared Raman spectroscopy and the histopathological analysis of atherosclerosis in human coronary arteries, Lasers in Surgery and Medicine 30 (2002), 290-297.

[32] N. Stone, C. Kendall, J. Smith, P. Crow and H. Barr, Raman spectroscopy for identification of epithelial cancers, Faraday Discussions 126 (2004), 141-157.

[33] N. Stone, M.C.H. Prieto, P. Crow, J. Uff and A.W. Ritchie, The use of Raman spectroscopy to provide an estimation of the gross biochemistry associated with urological pathologies, Analytical and Bioanalytical Chemistry 387 (2007), $1657-1668$.

[34] N. Stone, P. Stavroulaki, C. Kendall, M. Birchall and H. Barr, Raman spectroscopy for early detection of laryngeal malignancy: preliminary results, Laryngoscope 110 (2000), 1756-1763.

[35] S.K. Teh, W. Zheng, K.W. Ho, M. Teh, K.G. Yeoh and Z. Huang, Diagnostic potential of near-infrared Raman spectroscopy in the stomach: differentiating dysplasia from normal tissue, British Journal of Cancer 98 (2008), 457-465.

[36] H. Wold, Path models with latent variables: The NIPALS approach, in: Quantitative Sociology: International Perspectives on Mathematical and Statistical Model Building, H.M. Blalock, ed., Academic Press, New York, USA, 1975, pp. $307-357$. 


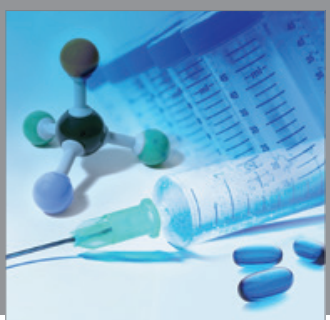

International Journal of

Medicinal Chemistry

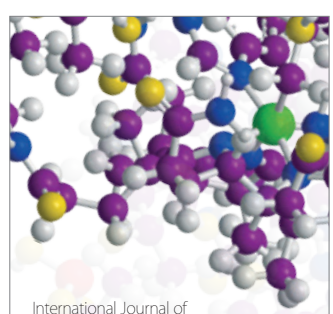

Carbohydrate Chemistry

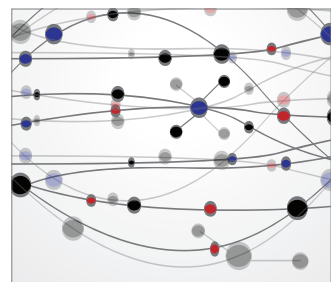

The Scientific World Journal
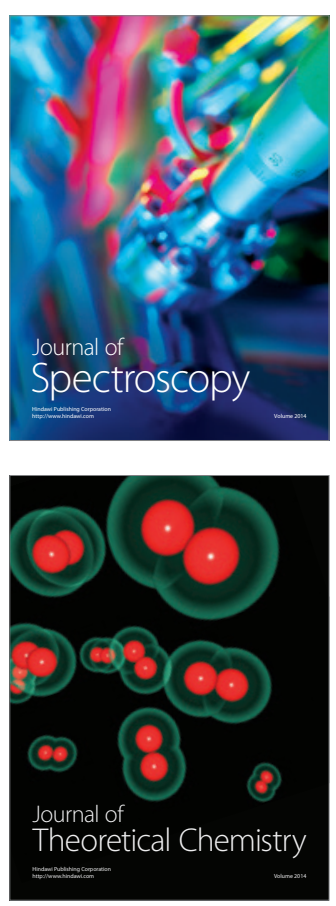
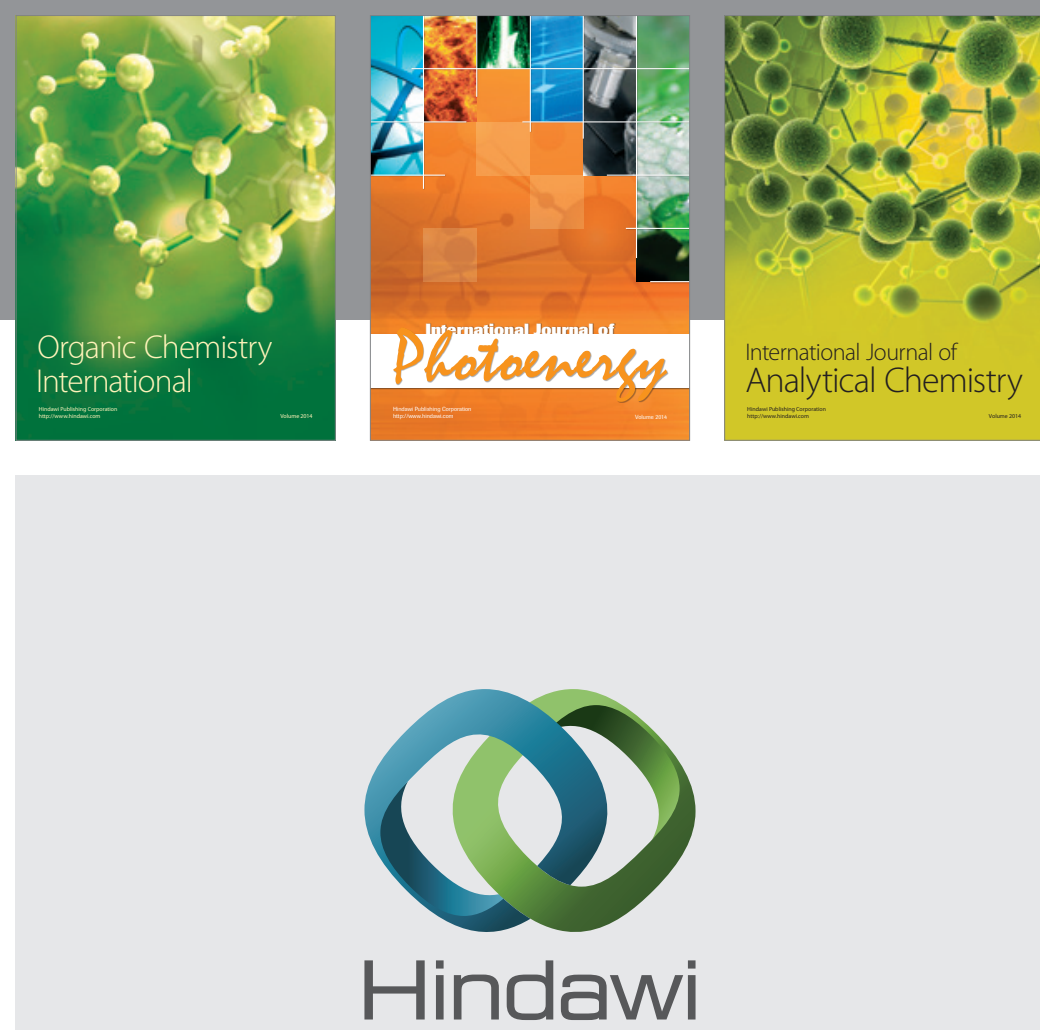

Submit your manuscripts at

http://www.hindawi.com
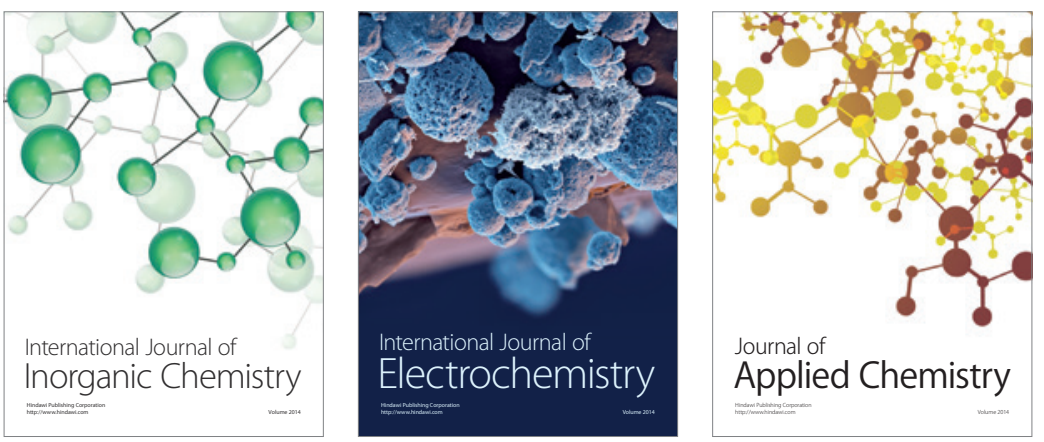

Journal of

Applied Chemistry
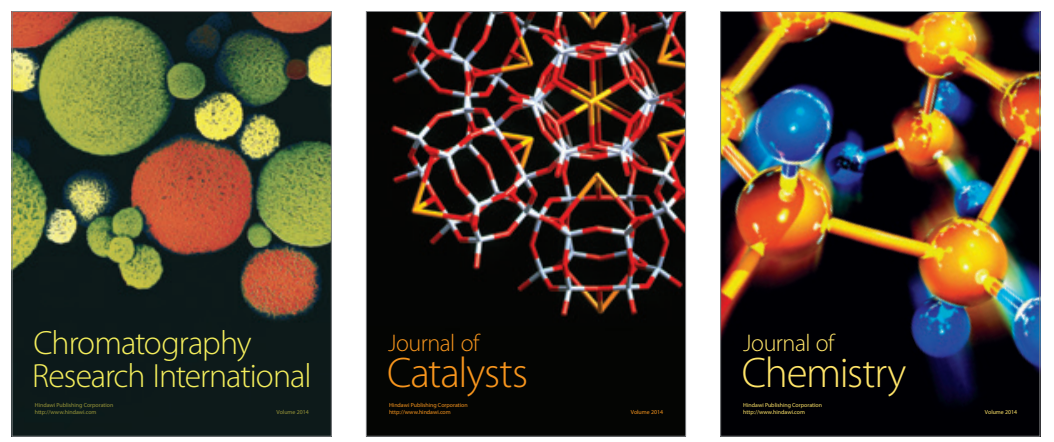
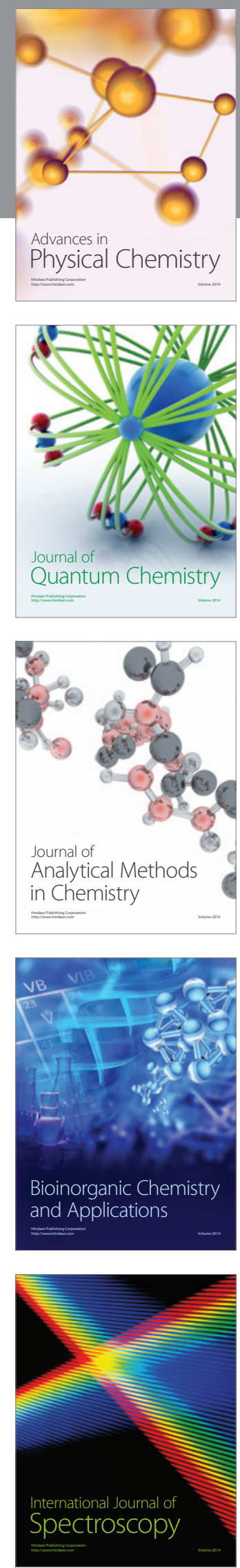